\author{
International Association \\ of Applied Mathematics and Mechanics \\ - Archive for Students -
}

\title{
Modeling the morphology evolution of organic solar cells
}

\author{
Kai Bergermann ${ }^{a, \star}$ \\ $a$ Technische Universität Chemnitz, Chemnitz, Germany \\ received 24.05.2019, accepted 05.08.2019, published 10.10.2019 \\ * corresponding author: kai.bergermann@s2017.tu-chemnitz.de \\ supervisor: Martin Stoll, TU Chemnitz, Germany \\ Uwe Streit, TU Chemnitz, Germany
}

\begin{abstract}
Organic solar cells present a promising alternative for the generation of solar energy at lower material and production costs compared to widely used siliconbased solar cells. The major drawback of organic solar cells currently is a lower rate of energy conversion. Thus many research projects aim to improve the achievable efficiency.

In this work a phase field model is used to mathematically describe the morphology evolution of the active layer composed of polymer as electron-donor and fullerene as electron-acceptor. The derivation of a chemical potential term and a surface energy term for the polymerfullerene solution using the Flory-Huggins theory forms the basis to employ the Cahn-Hilliard equation. After including several specifics of the application in this nonlinear partial differential equation of fourth order, an implementation of the model using the FEM solver software FEniCS provides some simulation results. Despite some simplifications compared to other models in the literature, the obtained results qualitatively match the simulation results from the literature.
\end{abstract}

Keywords: organic solar cells, mathematical modeling, Flory-Huggins theory, Cahn-Hilliard equation, FEniCS

\section{Introduction}

Since the enactment of the German Renewable Energy Law (Erneuerbare-Energien-Gesetz) in the year 2000 solar energy successively increased its share of the German energy mix from initially 0 to currently approximately $7 \%$ [5]. In comparison to widely spread silicon-based solar cells, organic solar cells present an alternative that could significantly lower material and production costs in the manufacturing process. As it has already been possible to print the active layer of organic solar cells on flexible and semi-transparent support material, this class of solar cells could also extend the area of application from currently mainly house roofs and flat areas to building fronts, car windows, backpacks, clothing, and many more. Additionally, while silicon-based solar cells can discharge toxic heavy metals into the environment at disposal, this is not an issue with organic solar cells [11].

Opposing the many advantages, long-term durability of organic solar cells is currently an issue, as are the achievable power conversion rates compared to siliconbased solar cells. While research on organic solar cells started with approximately $2 \%$ efficiency in the early 2000s, the efficiency record could be increased to approximately $10 \%$ until 2012 and ranges at $16 \%$ to date. 


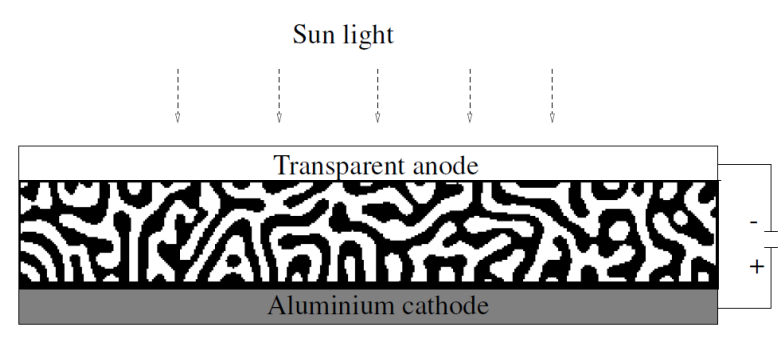

Figure 1: Schematic model of an organic solar cell [12, Fig. 1]

In comparison, silicon-based solar cells range at $25 \%$ efficiency since the mid 1990s. [2]

The class of organic solar cells considered in this work makes use of a polymer in the role of an electron-donor, a fullerene as an electron-acceptor and a solvent to initially form a homogeneous solution. During the manufacturing process this solution is spread onto a support material to form a thin film. At the top of the film, an evaporation process is initiated, in which the solvent is extracted from the film as it comes into contact with air. The result of this evaporation process is a phase separation in the remaining polymer and fullerene compound, forming rounded regions of pure components. The chemical reaction terminates when all solvent has evaporated. In the final architecture, the so formed active layer is framed by a transparent anode at the top surface and a reflective cathode at the bottom surface (Figure 1). Sun light entering the active layer through the anode then excites electrons in the electron-donor region such that they are carried to a phase boundary where the electron is transmitted to the electron-acceptor region. Through the conductive donor and acceptor regions the so emerging flow of electron and hole pairs is discharged as electricity. The reflective property of the cathode enables reflected sunrays to pass through the active layer again triggering electron transfers [12].

The mathematical description of the morphology evolution can be done by a phase field model [9, Chapter 10], that consists of a domain $\Omega \subseteq \mathbb{R}^{3}$ and scalar fields

$$
\phi_{p}, \phi_{f}, \phi_{s}: \Omega \times[0, T] \rightarrow[0,1] \subseteq \mathbb{R}
$$

representing the concentration of polymer, fullerene and solvent respectively at a given point in the domain at a given time in the interval $[0, T]$. The relation

$$
\phi_{p}+\phi_{f}+\phi_{s}=1
$$

applies for all $x \in \Omega$ and every $t \in[0, T]$.

Following the second law of thermodynamics, a thermodynamically closed system tends to minimize its free energy by maximizing its entropy. Thus the description of the chemical potential in the domain will play a key role in modeling the morphology evolution process.

The Ginzburg-Landau energy functional forms the basis for the Cahn-Hilliard equation and is given by

$$
\begin{aligned}
F\left(\phi_{p}, \phi_{f}, \phi_{s}\right)=\int_{\Omega}\left[f\left(\phi_{p}, \phi_{f}, \phi_{s}\right)+\frac{\epsilon_{p}}{2}\left|\nabla \phi_{p}\right|^{2}\right. \\
\left.+\frac{\epsilon_{f}}{2}\left|\nabla \phi_{f}\right|^{2}\right] \mathrm{d} \Omega+F_{s}\left(\phi_{p}, \phi_{f}, x\right),
\end{aligned}
$$

where $f\left(\phi_{p}, \phi_{f}, \phi_{s}\right)$ describes the inner energy density in the domain and $F_{s}\left(\phi_{p}, \phi_{f}, x\right)$ a surface energy correction term, which describes certain effects in molecular interaction energies occurring at the domains interface with the substrate material. The parameters $\epsilon_{p}, \epsilon_{f}$ represent (ideally small) interface parameters that place weight on the gradient terms. These gradient terms represent the Dirichlet energy, which penalizes strong concentration gradients and thus leads to rounded phase boundaries of minimal length in accordance with the energy minimization stated by the second law of thermodynamics. By relation (1) it is sufficient to describe the system by only two concentrations. Therefore the computational model in Section 4 only makes use of partial derivatives of the Ginzburg-Landau functional w.r.t. $\phi_{p}$ and $\phi_{f}$ and thus a concentration gradient for the solvent concentration does not need to be included in (2).

Section 2 will see to the modeling of the bulk energy term $f\left(\phi_{p}, \phi_{f}, \phi_{s}\right)$, following the Flory-Huggins theory. Section 3 models the surface energy term $F_{s}\left(\phi_{p}, \phi_{f}, x\right)$ to describe boundary effects. Section 4 then derives the Cahn-Hilliard equation, taking into account several specifics of the application of organic solar cells. After Section 5 showed the numerical implementation of the mathematical model, Section 6 finally presents results obtained by the implementation using the FEM solver software FEniCS.

\section{Flory-Huggins energy model}

As an implication of the first law of thermodynamics the Flory-Huggins theory is based on the approach

$$
\Delta G_{m}=\Delta H_{m}-T \Delta S_{m}
$$

to describe the inner energy $\Delta G_{m}$ of a thermodynamically closed polymer solution in terms of the enthalpy $\Delta H_{m}$ describing the heat of mixing and the entropy $\Delta S_{m}$ describing the thermodynamical relationship between the temperature and the energy content of the system.

The derivation of the inner energy makes use of a molecular lattice model and closely follows [7]. In terms 


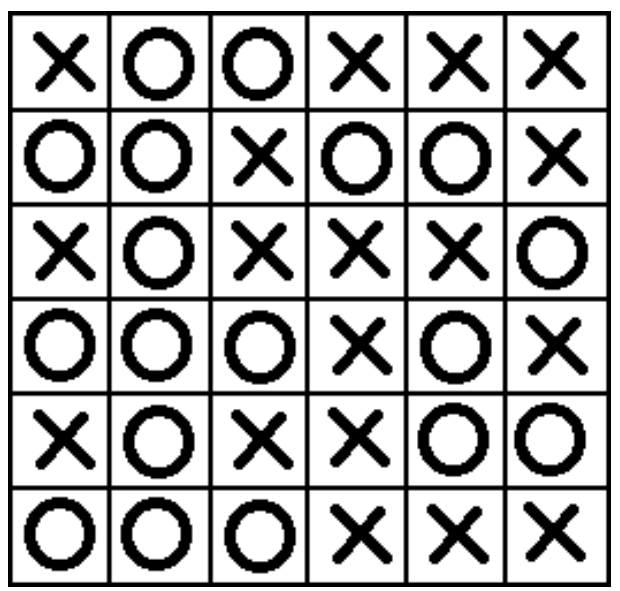

Figure 2: Example configuration of the molecular lattice

of notation, $\phi_{1}, \phi_{2}$ and $\phi_{3}$ will be used for general derivations before moving to the notation defined in the Introduction.

\subsection{Entropy}

To describe the entropy of a binary polymer solution, one considers an equidistant lattice, whose sites are exhaustively and randomly assigned with molecules of the two participating components, as illustrated in Figure 2. Thus there applies

$$
n=n_{1}+n_{2}
$$

for $n$ the total number of lattice sites, $n_{1}$ the number of molecules of type 1 and $n_{2}$ the number of molecules of type 2 . The number of distinct arrangements of molecules representing the number of possible micro states is given by

$$
\Omega:=\left(\begin{array}{c}
n \\
n_{1}
\end{array}\right)=\left(\begin{array}{c}
n \\
n_{2}
\end{array}\right)=\frac{n !}{n_{1} ! n_{2} !} .
$$

Now the Boltzmann relation is used to describe the thermodynamical probability of a macro state by

$$
\Delta S_{m}=k \ln (\Omega),
$$

where $k$ is Boltzmann's constant.

Inserting (3), continued application of the logarithm laws and usage of the Stirling approximation

$$
\ln (n !) \approx n \ln (n)-n
$$

leads to

$$
\Delta S_{m}=-k\left[n_{1} \ln \left(\frac{n_{1}}{n}\right)+n_{2} \ln \left(\frac{n_{2}}{n}\right)\right] .
$$

Introducing concentrations

$$
\phi_{1}=\frac{n_{1}}{n}, \quad \phi_{2}=\frac{n_{2}}{n}
$$

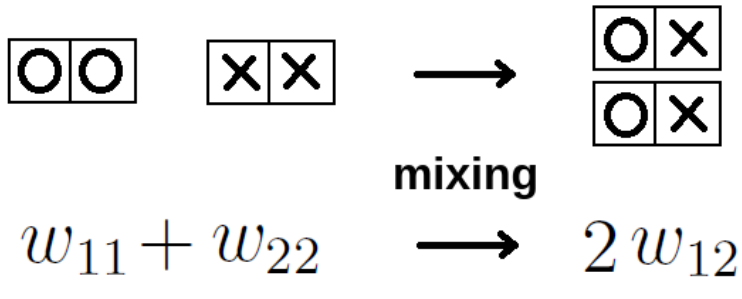

Figure 3: Molecular interaction energies of pure compounds in comparison with a mixture

and the volume

$$
V=n_{1} v_{1}+n_{2} v_{2}
$$

allowing the two sorts of molecules to have different volumes $v_{1}$ and $v_{2}$, instead of making the restrictive assumption of equidistance of the lattice, the entropy can be expressed by

$$
\Delta S_{m}=-k V\left[\frac{\phi_{1}}{v_{1}} \ln \left(\phi_{1}\right)+\frac{\phi_{2}}{v_{2}} \ln \left(\phi_{2}\right)\right] .
$$

A straightforward generalization extends this description to ternary systems consisting of three concentrations $\phi_{1}, \phi_{2}$ and $\phi_{3}$ :

$$
\Delta S_{m}=-k V\left[\frac{\phi_{1}}{v_{1}} \ln \left(\phi_{1}\right)+\frac{\phi_{2}}{v_{2}} \ln \left(\phi_{2}\right)+\frac{\phi_{3}}{v_{3}} \ln \left(\phi_{3}\right)\right] .
$$

Adapting the notation to [12], moving from molecular volumes to molar volumes (leading to the replacement of $k$ by Avogadro's gas constant $R$ [10]) and introducing a reference volume $V^{s}$ as well as a degree of polymerization

$$
N_{i}=\frac{v_{i}}{V^{s}}, \quad i \in\{p, f, s\}
$$

the final version of the entropy reads

$$
\Delta S_{m}=\frac{R}{V^{s}}\left[\frac{\phi_{p}}{N_{p}} \ln \left(\phi_{p}\right)+\frac{\phi_{f}}{N_{f}} \ln \left(\phi_{f}\right)+\frac{\phi_{s}}{N_{s}} \ln \left(\phi_{s}\right)\right] .
$$

\subsection{Enthalpy}

The enthalpy describes the total molecular interaction energy in a given configuration on the lattice. To do so the direct neighbors of any given molecule are considered. Interaction energies to more distant molecules can be neglected as molecular interaction energies rapidly decrease as a function of distance. Now let $w_{11}$ describe the molecular interaction energy between two molecules of type $1, w_{22}$ that of two molecules of type 2 and $w_{12}$ that of two neighboring pairs of different type. The energetic difference between one neighboring pair in a mixture in comparison to the pure compounds can then be expressed as

$$
\Delta w_{12}=w_{12}+\frac{1}{2}\left(w_{11}+w_{22}\right)
$$


as it is shown in Figure 3.

The interaction energy of the whole system thus is the sum of $\Delta w_{12}$ over all neighboring pairs of different type. Denoting this number with $p_{12}$ lets one define the enthalpy as

$$
\Delta H_{m}=\Delta w_{12} p_{12} .
$$

Now interpreting $\frac{n_{2}}{n}$ as the probability to find a molecule of type 2 at a randomly picked neighboring site of any molecule of type 1 and defining $z$ as the global number of neighbors of each molecule (which is a simplification as boundary sites are not treated separately), one can sum up all heterogeneous neighboring pairs as

$$
p_{12}=n_{1} z \frac{n_{2}}{n} .
$$

The Flory-Huggins interaction parameter is then defined as

$$
\chi_{12}=\frac{z \Delta w_{12}}{k T} .
$$

Inserting everything into (7) gives

$$
\Delta H_{m}=k T \chi_{12} n_{1} \frac{n_{2}}{n} .
$$

Again substituting the concentrations (4) and using a general description of the volume (5) this equation becomes

$$
\Delta H_{m}=\frac{k T \chi_{12} \phi_{1} V \phi_{2}}{v_{1}} .
$$

Moving towards a ternary system, as needed for the organic solar cell model, one needs to consider three possible combinations of heterogeneous neighboring pairs with $\Delta w_{12}, \Delta w_{13}$ and $\Delta w_{23}$ analogously defined as in (6). The same arguments, the adoption of the notation of [12] and again the introduction of a reference volume $V^{s}$ lead to

$$
\Delta H_{m}=\frac{R T}{V^{s}}\left[\chi_{p f} \phi_{p} \phi_{f}+\chi_{p s} \phi_{p} \phi_{s}+\chi_{f s} \phi_{f} \phi_{s}\right] .
$$

All together for binary mixtures the free energy is given by

$$
\begin{aligned}
\Delta G_{m} & =\Delta H_{m}-T \Delta S_{m} \\
& =k T V\left[\frac{\chi_{12} \phi_{1} \phi_{2}}{v_{1}}+\frac{\phi_{1}}{v_{1}} \ln \left(\phi_{1}\right)+\frac{\phi_{2}}{v_{2}} \ln \left(\phi_{2}\right)\right] .
\end{aligned}
$$

For ternary systems the energy density $f\left(\phi_{p}, \phi_{f}, \phi_{s}\right)$ in (2) has been found to be

$$
\begin{aligned}
f\left(\phi_{p}, \phi_{f}, \phi_{s}\right)= & \frac{R T}{V^{s}}\left[\frac{\phi_{p}}{N_{p}} \ln \left(\phi_{p}\right)+\frac{\phi_{f}}{N_{f}} \ln \left(\phi_{f}\right)+\frac{\phi_{s}}{N_{s}} \ln \left(\phi_{s}\right)\right. \\
& \left.+\chi_{p f} \phi_{p} \phi_{f}+\chi_{p s} \phi_{p} \phi_{s}+\chi_{f s} \phi_{f} \phi_{s}\right] .
\end{aligned}
$$
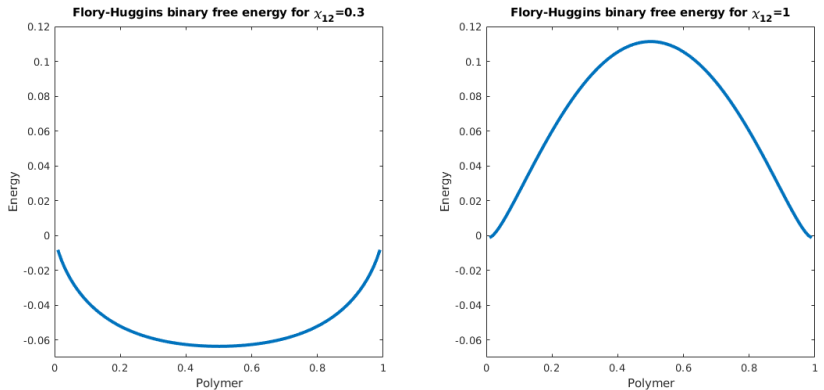

Figure 4: Binary free energy of a polymer-solvent compound for Flory-Huggins parameters $\chi_{12}=0.3$ and $\chi_{12}=1$

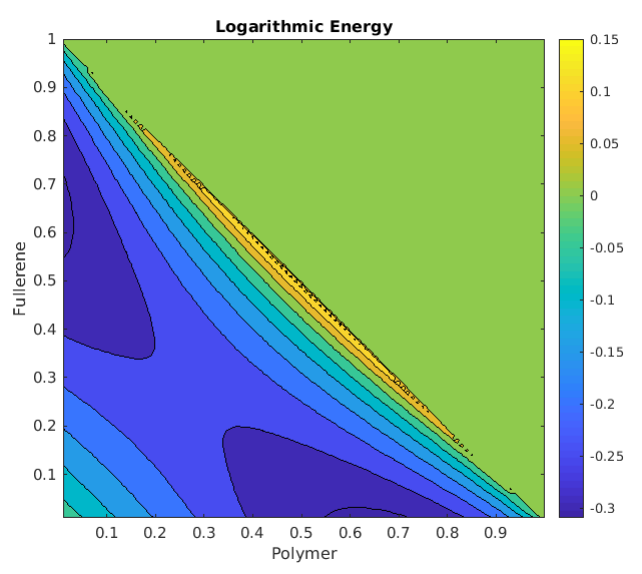

Figure 5: Contour plot of the free energy $f\left(\phi_{p}, \phi_{f}, \phi_{s}\right)$ of the ternary system consisting of polymer, fullerene and solvent

\subsection{Interpretation}

First considering the binary formulation (9) one can interpret the Flory-Huggins interaction parameter as a measure of the instability of a polymer solution. Figure 4 shows the free energy in a polymer-solvent compound as a function of the polymer concentration. For a Flory-Huggins interaction parameter of $\chi_{12}=0.3$ the minimum of the free energy is located at $\phi_{1}=0.5$. Due to the relation

$$
\phi_{1}+\phi_{2}=1
$$

in the binary case it follows $\phi_{2}=0.5$ and thus the homogeneous mixture is the energetically most favorable situation and any compound will strive towards a mixed state. For $\chi_{12}=1$ on the other hand, the minima of the free energy are located at $\phi_{1}=0$ and $\phi_{1}=1$, so in this situation the phase separation into pure polymer and pure solvent regions will be energetically optimal. The reason for this behavior can be traced back to the molecular interaction energies in (6).

In the application of organic solar cells the two conducting materials polymer and fullerene are character- 
ized by a large Flory-Huggins interaction parameter of approximately $\chi_{p f}=1$ [12]. So to create the homogeneous mixture, that is needed as initial condition for the manufacturing process, a solvent that favors mixing with the two components is added. This typically leads to interaction parameters of approximately $\chi_{p s}=$ $\chi_{f s}=0.3$ [12]. Figure 5 shows a plot of the free energy in this ternary situation as a function of the polymer and fullerene concentrations. The solvent concentration is implicitly determined by the relation (1). Here the global energetic minima are located at $\left(\phi_{p}=\frac{2}{3}, \phi_{f}=0, \phi_{s}=\frac{1}{3}\right)$, as well as at $\left(\phi_{p}=0, \phi_{f}=\frac{2}{3}, \phi_{s}=\frac{1}{3}\right)$. However, the manufacturing process of organic solar cells is characterized by the evaporation process at the top surface that continuously reduces the solvent content in the system. As the production process evolves and the solvent concentration $\phi_{s}$ goes to 0 , the system converges to the binary situation in Figure 4 with $\chi_{p f}=1$ (characterized by the linear connection of the points $\left(\phi_{p}=1, \phi_{f}=0\right)$ and $\left(\phi_{p}=0, \phi_{f}=1\right)$ in Figure 5) leaving the continued phase separation into pure regions of polymer and fullerene as the energetically favorable state that can be observed in the real manufacturing process.

\section{Surface energy}

The need for a surface energy term correcting the description of the inner energy derived in the last section can both be motivated phenomenologically, as well as theoretically.

The source [8, Chapter 5] phenomenologically describes, that the formation of a different morphology in the region of an interface with another material can have a positive impact on the energy balance of the system. For this phenomenon to occur, the molecular interaction energies between at least one component of the polymer mixture and the interfacing material must be smaller than the interaction energies within the solution itself.

Theoretically speaking in terms of the molecular lattice model, Section 2.2 made the assumption that each lattice site has a fixed number of neighboring cells. This is of course untrue for molecules on a boundary site.

The following derivation of the surface energy term $F_{s}\left(\phi_{p}, \phi_{f}, x\right)$, which is added to the inner energy in the Ginzburg-Landau energy functional (2) follows [8, Chapter 5]. Again, a binary system is considered first and only one variable $\phi$ is used, leaving the other concentration as $1-\phi$.

The creation of a surface in terms of the molecular lattice model can be thought of as cutting through the bulk of the solution and accounting for the molecular

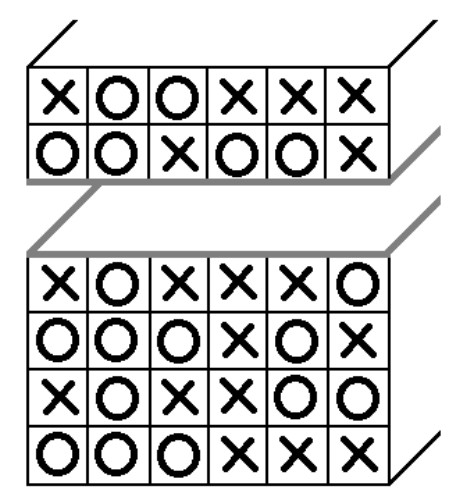

Figure 6: Generating a surface of a polymer solution by cutting through the bulk region

interaction energies eliminated by doing so. (To be precise, one should probably replace the removed neighboring molecules' energies by those of the interfacing molecules instead of eliminating them, but this procedure is not carried out in this theory.)

Considering one boundary site in the situation of Figure 6 and interpreting the concentrations $\phi$ and $(1-\phi)$ as probabilities to find a molecule of the corresponding type at a randomly picked site, this leaves four combinations of formerly neighboring pairs:

1. both were of type 1. Probability: $\phi^{2}$

2. both were of type 2 . Probability: $(1-\phi)^{2}$

3. two combinations of different type. Probability: $\phi(1-\phi)$

As the given probabilities sum up to 1 it is reasonable to interpret the following expression as expectation value of the molecular interaction energy lost for one formerly neighboring pair:

$$
\mathbb{E}(w)=\left[\phi^{2} w_{11}+(1-\phi)^{2} w_{22}+2 \phi(1-\phi) w_{12}\right] .
$$

Multiplication of this expectation with the number of cut bonds $z^{\prime}$ and a lattice parameter $\frac{b}{2}$ [8, Chapter 5], lets the surface energy density be written as

$f_{\mathcal{s}}(\phi)=\frac{z^{\prime} b}{2}\left[\phi^{2} w_{11}+\left(1-2 \phi+\phi^{2}\right) w_{22}+2\left(\phi-\phi^{2}\right) w_{12}\right]^{1}$

Rewriting the Flory-Huggins interaction parameter (8) in terms of $w_{12}$, rearranging (11) and omitting the constant term leads to

$$
f_{s}(\phi)=g \phi+h \phi^{2}
$$

with parameters $g$ and $h$ depending on the Flory-Huggins parameter as well as several lattice parameters.

\footnotetext{
${ }^{1}$ Equation (11) slightly differs from [8, Equation (5.1.4)] as it corrects a typo made in the original work
} 
The extension to the ternary system of polymer, fullerene and solvent is achieved by simply adding up (12) for polymer and fullerene and multiplying each one of them by a space-dependent function $p_{p}(x)$ and $p_{f}(x)$ respectively, which are defined for all points $x \in \Gamma_{b}$ on the film's interface with the substrate material $\Gamma_{b}$. Thus all together the surface energy density is given by

$$
\begin{aligned}
f_{s}\left(\phi_{p}, \phi_{f}, x\right)=p_{p}(x)\left(g_{p} \phi_{p}+h_{p} \phi_{p}^{2}\right) & \\
& +p_{f}(x)\left(g_{f} \phi_{f}+h_{f} \phi_{f}^{2}\right) .
\end{aligned}
$$

Integration over the surface $\Gamma_{b}$ and multiplication with the usual factor gives

$$
F_{s}\left(\phi_{p}, \phi_{f}, x\right)=-\frac{R T}{V^{s}} \int_{\Gamma_{b}} f_{\mathcal{s}}\left(\phi_{p}, \phi_{f}, x\right) \mathrm{d} \Gamma_{b}
$$

as the total surface energy to be used in the GinzburgLandau functional (2).

\section{The Cahn-Hilliard equation}

The Cahn-Hilliard equation is a non-linear partial differential equation of fourth order that is often used to model phase separation processes in e.g., metals, polymers or glasses. Its solution $\phi$ is a scalar field on a domain $\Omega \subseteq \mathbb{R}^{3}$. Additionally, the chemical potential $\mu$ plays a key role. It is given as the partial derivative of the Ginzburg-Landau functional (2) w.r.t. $\phi$.

Among other possibilities the Cahn-Hilliard equation can be derived as a gradient flow using the mass conservation law [3]:

$$
\frac{\partial \phi}{\partial t}=-\nabla \cdot \mathbf{J},
$$

which corresponds to Fick's second law with the definition of $\mathbf{J}$ as

$$
\mathbf{J}=-M(\phi) \nabla \mu,
$$

where $M(\phi)$ is a mobility function that generally depends on the concentration $\phi$. For simplicity it is assumed to be constant in this work. Non-constant mobilities have not been studied in much detail for the problem discussed here. In the Cahn-Hilliard case we refer to [6], where it is shown that both the model complexity and the numerical solution complexity is significantly increased.

Inserting $\mu=\frac{\partial F}{\partial \phi}$ for the binary situation and omitting the surface energy term for now, the binary CahnHilliard equation becomes

$$
\frac{\partial \phi}{\partial t}=\nabla \cdot M \nabla\left(\frac{\partial f(\phi)}{\partial \phi}-\epsilon^{2} \nabla^{2} \phi\right) \quad \text { in } \Omega \times[0, T] .
$$

Rewriting this in a decoupled way, i.e., pulling the chemical potential $\mu$ into a separate equation leaving two equations of second order and adding homogeneous Neumann boundary conditions to account for thermodynamic closure, as well as initial conditions, one obtains

$$
\begin{array}{rlrl}
\frac{\partial \phi}{\partial t} & =\nabla \cdot M \nabla \mu & & \text { in } \Omega \times[0, T], \\
\mu & =\frac{\partial f(\phi)}{\partial \phi}-\epsilon^{2} \nabla^{2} \phi & & \text { in } \Omega \times[0, T], \\
M \nabla \mu \cdot \mathbf{n} & =0 & & \text { on } \Gamma \times[0, T], \\
\phi(x, 0) & =\phi^{0}(x) & \text { in } \Omega .
\end{array}
$$

For a general ternary system the same initial boundary value problem (IBVP) is written for two of the three components. The notation $\phi_{p}, \phi_{f}$ and $\phi_{s}$ is used in preparation for the IBVP for organic solar cells:

$$
\begin{array}{rlrl}
\frac{\partial \phi_{p}}{\partial t} & =\nabla \cdot M_{p} \nabla \mu_{p} & & \text { in } \Omega \times[0, T], \\
\frac{\partial \phi_{f}}{\partial t} & =\nabla \cdot M_{f} \nabla \mu_{f} & & \text { in } \Omega \times[0, T], \\
\mu_{p} & =\frac{\partial f}{\partial \phi_{p}}-\epsilon^{2} \nabla^{2} \phi_{p} & & \text { in } \Omega \times[0, T], \\
\mu_{f} & =\frac{\partial f}{\partial \phi_{f}}-\epsilon^{2} \nabla^{2} \phi_{f} & & \text { in } \Omega \times[0, T], \\
M_{p} \nabla \mu_{p} \cdot \mathbf{n} & =0 & & \text { on } \Gamma \times[0, T], \\
M_{f} \nabla \mu_{f} \cdot \mathbf{n} & =0 & & \text { on } \Gamma \times[0, T], \\
\phi_{p}(x, 0) & =\phi_{p}^{0}(x) & \text { in } \Omega, \\
\phi_{f}(x, 0) & =\phi_{f}^{0}(x) & \text { in } \Omega,
\end{array}
$$

with $\mu_{p}=\frac{\partial F}{\partial \phi_{p}}$ and $\mu_{f}=\frac{\partial F}{\partial \phi_{f}}$ denoting the chemical potential of polymer and fullerene respectively. A sensible relationship between the two sets of equations is ensured by the mutually used potential term $f\left(\phi_{p}, \phi_{f}, \phi_{s}\right)$. Without it, the solution of (14) would contain two independent solutions without any constraints like (1).

To model the manufacturing process of organic solar cells, (14) is now extended by some key physical properties of the application. First, the partial derivatives of the surface energy terms, introduced in Section 3, are added to the chemical potentials $\mu_{p}$ and $\mu_{f}$ using the Heaviside function

$$
H(h)= \begin{cases}0, & \text { for } h \leq 0, \\ 1, & \text { for } h>0\end{cases}
$$

to activate this term on the lower bound only [12].

Second, following [12], the assumption of thermodynamic closure is no longer true due to the evaporation process of the solvent on the upper boundary. To account for this, the flux of chemical potential out of the 
system can be described by a negative solvent flux proportional to the current solvent concentration

$$
h_{\mu_{s}}=-J_{s}^{a} \phi_{s} .
$$

Now a negative flux of solvent is equivalent to a positive flux of polymer and fullerene and thus one can write

$$
h_{\mu_{p}}=J_{s}^{a} \phi_{p} \quad \text { and } \quad h_{\mu_{f}}=J_{s}^{a} \phi_{f},
$$

which can be incorporated in the IBVP by Neumann boundary conditions

$$
\begin{array}{ll}
M_{p} \nabla \mu_{p} \cdot \mathbf{n}=h_{\mu_{p}} & \text { on } \Gamma_{t} \times[0, T], \\
M_{f} \nabla \mu_{f} \cdot \mathbf{n}=h_{\mu_{f}} & \text { on } \Gamma_{t} \times[0, T],
\end{array}
$$

where $\Gamma_{t}$ denotes the top boundary of $\Omega$.

While [12] models the flux of the solvent concentration $\phi_{s}$ by a volume-dependent function, the introduction of a time-dependent height function and a coordinate transformation to account for the change in domain size due to the evaporation, here, the flux of $\phi_{s}$ is treated analogously to the flux of the chemical potential $\mu_{s}$. So the flux of solvent concentration out of the system corresponds to a flux of polymer and fullerene into the system, leading to

$$
g_{\phi_{p}}=J_{s}^{a} \phi_{p} \quad \text { and } \quad g_{\phi_{f}}=J_{s}^{a} \phi_{f},
$$

such that boundary conditions

$$
\begin{aligned}
\epsilon^{2} \nabla \phi_{p} \cdot \mathbf{n} & =g_{\phi_{p}} & & \text { on } \Gamma_{t} \times[0, T], \\
\epsilon^{2} \nabla \phi_{f} \cdot \mathbf{n} & =g_{\phi_{f}} & & \text { on } \Gamma_{t} \times[0, T] .
\end{aligned}
$$

are formulated.

The homogeneous Neumann boundary conditions

$$
\begin{aligned}
M_{i} \nabla \mu_{i} \cdot \mathbf{n} & =0 & & \text { on } \Gamma \backslash \Gamma_{t} \times[0, T] \\
\epsilon^{2} \nabla \phi_{i} \cdot \mathbf{n} & =0 & & \text { on } \Gamma \backslash \Gamma_{t} \times[0, T]
\end{aligned}
$$

for $i \in\{p, f\}$ are now not explicitly stated in the IBVP any more. The stochastic noise terms $\xi_{p}$ and $\xi_{f}$ included in [12] to model white noise in the compound are omitted for simplicity.

All together the strong form of the IBVP reads

$$
\begin{aligned}
\frac{\partial \phi_{p}}{\partial t} & =\nabla \cdot M_{p} \nabla \mu_{p} & & \text { in } \Omega \times[0, T], \\
\frac{\partial \phi_{f}}{\partial t} & =\nabla \cdot M_{f} \nabla \mu_{f} & & \text { in } \Omega \times[0, T], \\
\mu_{p} & =\frac{\partial f}{\partial \phi_{p}}-\epsilon^{2} \nabla^{2} \phi_{p}+(1-H(h)) \frac{\partial f_{s}}{\partial \phi_{p}} & & \text { in } \Omega \times[0, T], \\
\mu_{f} & =\frac{\partial f}{\partial \phi_{f}}-\epsilon^{2} \nabla^{2} \phi_{f}+(1-H(h)) \frac{\partial f_{s}}{\partial \phi_{f}} & & \text { in } \Omega \times[0, T], \\
M_{p} \nabla \mu_{p} \cdot \mathbf{n} & =h_{\mu_{p}} & & \text { on } \Gamma_{t} \times[0, T],
\end{aligned}
$$

$$
\begin{array}{rlrl}
M_{f} \nabla \mu_{f} \cdot \mathbf{n} & =h_{\mu_{f}} & & \text { on } \Gamma_{t} \times[0, T], \\
\epsilon^{2} \nabla \phi_{p} \cdot \mathbf{n} & =g_{\phi_{p}} & & \text { on } \Gamma_{t} \times[0, T], \\
\epsilon^{2} \nabla \phi_{f} \cdot \mathbf{n} & =g_{\phi_{f}} & & \text { on } \Gamma_{t} \times[0, T], \\
\phi_{p}(x, 0) & =\phi_{p}^{0}(x) & \text { in } \Omega, \\
\phi_{f}(x, 0) & =\phi_{f}^{0}(x) & \text { in } \Omega .
\end{array}
$$

\section{Numerical implementation}

Now, introducing the $\theta$-rule, as presented in [4] for $\theta=1$, to discretize the time derivatives as

$$
\begin{aligned}
\frac{\partial \phi_{i}}{\partial t} & =\frac{\phi_{i}\left(t_{j+1}\right)-\phi_{i}\left(t_{j}\right)}{\Delta t} \\
& =(1-\theta) \nabla \cdot M_{i} \nabla \mu_{i}\left(t_{j}\right)+\theta \nabla \cdot M_{i} \nabla \mu_{i}\left(t_{j+1}\right) \\
& =: M_{i} \nabla \cdot \nabla \mu_{i}\left(t_{j+\theta}\right)
\end{aligned}
$$

for concentrations $\phi_{i}\left(t_{j}\right), i \in\{p, f\}$ at time $t_{j} \in[0, T]$ and testing (15), one obtains the weak form as

$$
\begin{gathered}
\int_{\Omega} \frac{\phi_{p}\left(t_{j+1}\right)-\phi_{p}\left(t_{j}\right)}{\Delta t} q_{1} \mathrm{~d} x \\
+\int_{\Omega}\left(M_{p} \nabla \mu_{p}\left(t_{j+\theta}\right)\right) \nabla q_{1} \mathrm{~d} x-\int_{\Gamma_{t}} h_{\mu_{p}\left(t_{j+\theta}\right)} q_{1} \mathrm{~d} s=0
\end{gathered}
$$

$$
\begin{gathered}
\int_{\Omega} \frac{\phi_{f}\left(t_{j+1}\right)-\phi_{f}\left(t_{j}\right)}{\Delta t} q_{2} \mathrm{~d} x \\
+\int_{\Omega}\left(M_{f} \nabla \mu_{f}\left(t_{j+\theta}\right)\right) \nabla q_{2} \mathrm{~d} x-\int_{\Gamma_{t}} h_{\mu_{f}\left(t_{j+\theta}\right)} q_{2} \mathrm{~d} s=0 \\
\int_{\Omega} \mu_{p}\left(t_{j+1}\right) v_{1} \mathrm{~d} x-\int_{\Omega} \frac{\partial f}{\partial \phi_{p}\left(t_{j+1}\right)} v_{1} \mathrm{~d} x \\
-\epsilon_{\Omega} \epsilon^{2} \nabla \phi_{p}\left(t_{j+1}\right) \cdot \nabla v_{1} \mathrm{~d} x+\int_{\Gamma_{t}} g_{\phi_{p}\left(t_{j+1}\right)} v_{1} \mathrm{~d} s \\
-\int_{\Gamma_{b}} \frac{\partial f_{s}}{\partial \phi_{p}\left(t_{j+1}\right)} v_{1} \mathrm{~d} s=0
\end{gathered}
$$

$$
\begin{gathered}
\int_{\Omega} \mu_{f}\left(t_{j+1}\right) v_{2} \mathrm{~d} x-\int_{\Omega} \frac{\partial f}{\partial \phi_{f}\left(t_{j+1}\right)} v_{2} \mathrm{~d} x \\
-\int_{\Omega} \epsilon^{2} \nabla \phi_{f}\left(t_{j+1}\right) \cdot \nabla v_{2} \mathrm{~d} x+\int_{\Gamma_{t}} g_{\phi_{f}\left(t_{j+1}\right)} v_{2} \mathrm{~d} s \\
-\int_{\Gamma_{b}} \frac{\partial f_{s}}{\partial \phi_{f}\left(t_{j+1}\right)} v_{2} \mathrm{~d} s=0
\end{gathered}
$$




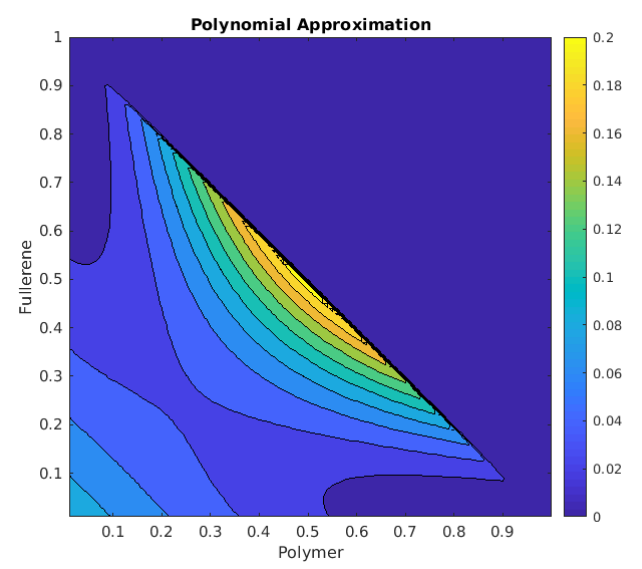

Figure 7: Numerically stable polynomial approximation of the logarithmic potential term Figure 5

with test functions $q_{1}, q_{2}, v_{1}$ and $v_{2}$ taken in the appropriate spaces and $\Gamma_{b}$ denoting the bottom boundary of $\Omega$.

The numerical implementation was done by extending the Cahn-Hilliard demo version of the FEniCS project, a python-based FEM solver software [1]. In this implementation the potential term (10) had to be replaced by a polynomial approximation for numerical stability's sake. Usage of the logarithmic potential caused the algorithm to terminate whenever the solution at any point in time dropped slightly below 0 at some spatial point. The polynomial

$$
f\left(\phi_{p}, \phi_{f}, \phi_{s}\right)=3.5 \phi_{p}^{2} \phi_{f}^{2}+0.1 \phi_{s}^{2}
$$

displayed in Figure 7 was found to qualitatively approximate (10), displayed in Figure 5, well. As negative concentrations just get penalized in (17) instead of causing run time errors, when passing negative values to the logarithm, it keeps the program up and running. The fact that Figure 7 takes only positive values, while Figure 5 is not an issue as constants will be omitted in the partial derivations of the potential term.

\section{Results}

The simulations were carried out using the finite element software FEniCS version 2017.2.0. Beside general observations, the focus in this section lies on the effect of the surface energy term derived in Section 3.

The model includes several material and reaction parameters. Following [12] for the chemical potential (10) the Flory-Huggins parameters $\chi_{p f}=1$ and $\chi_{p s}=\chi_{f s}=$ 0.3 were used. The representation of (17) was chosen to match these material parameters. According to [12], the parameters $h_{i}$ in (13) are negligibly small compared to
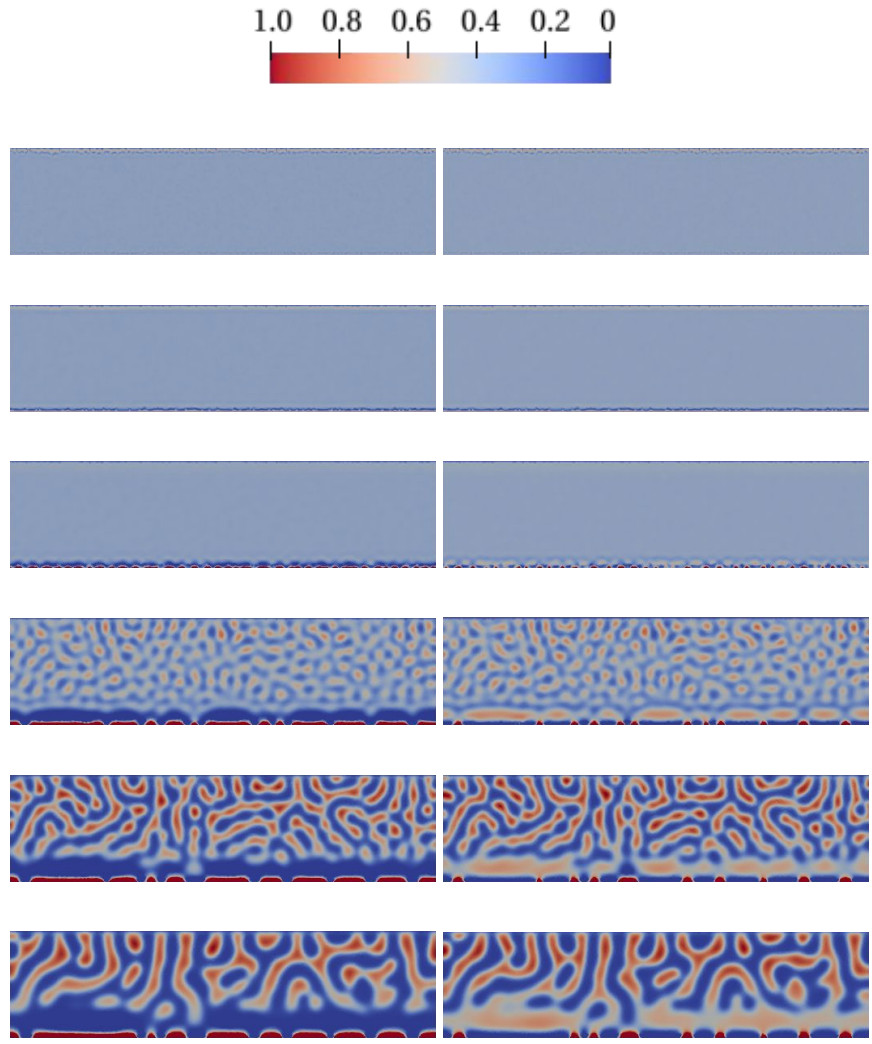

Figure 8: Morphology evolution for equal preference of polymer and fullerene at the substrate, i.e., $p_{p}(x)=p_{f}(x)=1$ for all $x \in \Gamma_{b}$, using parameters $g_{p}=g_{f}=0.1$

$g_{i}$. Thus, if not stated differently, these parameters have been set to $g_{i}=0.1$ and $h_{i}=0$ for $i \in\{p, f\}$ respectively. The flux parameter $J_{s}^{a}$ was found to generate a good concentration evolution at $J_{s}^{a}=5 \cdot 10^{4}$, raising the combined fraction of $\phi_{p}+\phi_{f}$ to approximately 1 within the time frame that it takes the chemical reaction to form the typical morphology. The initial conditions were set to $\phi_{p}=\phi_{f}=0.35 \pm 0.01$ with a random shift of up to $1 \%$ of the total concentration to model slight deviations from perfect homogeneity in the solution. The interface parameters were chosen $\epsilon_{p}=\epsilon_{f}=10^{-3}$, while the time stepping parameter $\theta=0.5$ (Crank-Nicolson) achieved the best results. To take care of the non-linearity of the equation due to the potential terms $f$ and $f_{s}$, a Newton solver is invoked in every time step [4]. The time step size $\Delta t$ was initialized at $\Delta t=10^{-5}$ and subsequently raised to $\Delta t=10^{-4}$ and $\Delta t=10^{-3}$ after 100 time steps respectively to account for the deceleration of the chemical reaction speed.

For the spatial discretization of the 2D calculations, linear triangular finite elements were used. FEniCS' built-in mesh generator created an unstructured grid of 14,390 elements. For the 3D calculations, a total number of 127,776 linear tetrahedral elements were used for dis- 

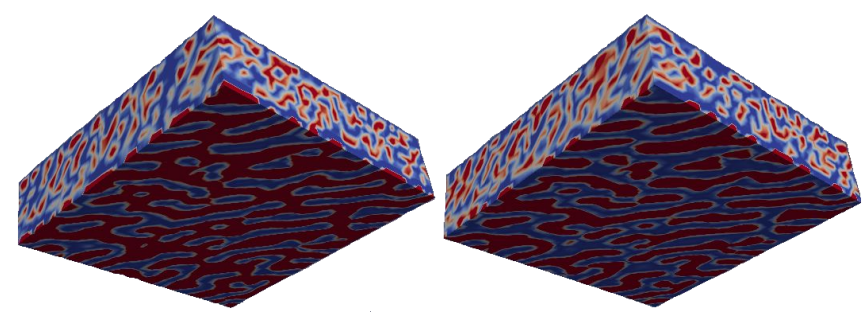

Figure 9: Lower boundary of a 3D simulation with equal preference of polymer and fullerene at the substrate, i.e., $p_{p}(x)=p_{f}(x)=1$ for all $x \in \Gamma_{b}$, using parameters $g_{p}=g_{f}=0.1$

cretization. As also mentioned in [12] it was found that quadratic or cubic basis functions do not significantly improve the quality of the results.

Figure 8 shows the morphology evolution with an equal preference of polymer and fullerene at the bottom surface, which corresponds to $p_{p}(x)=p_{f}(x)=1$ for all $x \in \Gamma_{b}$ in (13). The left hand side shows the polymer concentration $\phi_{p}$ and the right hand side the fullerene concentration $\phi_{f}$, while the solvent concentration is given by

$$
\phi_{s}=1-\phi_{p}-\phi_{f}
$$

While the simulation starts with an approximately homogeneous solution, small regions of pure polymer and fullerene are formed at the substrate in the beginning of the manufacturing process. Above this boundary region, a layer of fullerene is formed. At $t=0.03$ in picture 4 the phase separation process in the bulk region sets in, first generating small rounded regions of pure polymer and fullerene concentrations, followed by a coarsening into larger regions, forming the typical morphology of the active layer of organic solar cells. At $t=0.2$ the reaction speed becomes very slow and at $t=0.3$ (Picture 6 in Figure 8) the system has come close to the equilibrium configuration. There is no significant change in morphology between $t=0.3$ and $t=0.5$.

While Figure 8 seems to gather more polymer at the bottom boundary in comparison with fullerene, a 3D simulation in Figure 9 illustrates the 2 dimensional substrate to arrange in round islands of roughly equal proportions, similar to the bulk region of the $2 \mathrm{D}$ simulation. The degrees of freedom, i.e., the possible fineness of the spatial discretization and thus the accuracy of the results in Figure 9 was limited by the memory capacity of the single processor used for the simulation.

Setting $p_{p}(x)=1$ and $p_{f}(x)=0$ for all $x \in \Gamma_{b}$ in (13) instead and thus creating a preference of polymer at the entire substrate, leads to the formation of a layer of pure polymer at the substrate, followed by a thicker fullerene region, when moving upwards, see Figure 10.
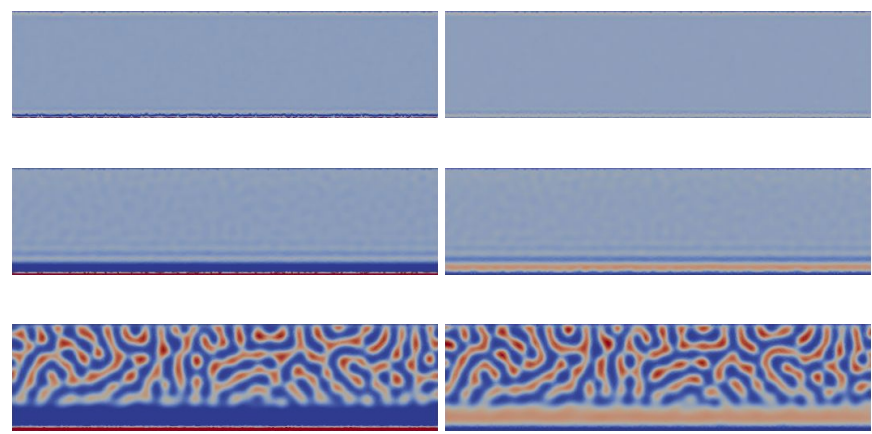

Figure 10: Morphology evolution for the preference of polymer at the substrate, i.e., $p_{p}(x)=1$, $p_{f}(x)=0$, using $g_{p}=0.1$

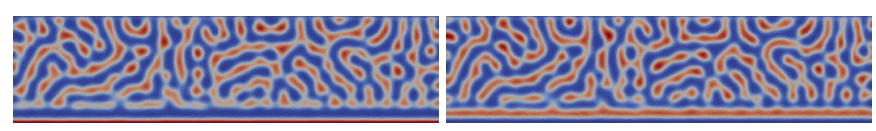

Figure 11: Morphology evolution for a weaker preference of polymer at the substrate, i.e., $p_{p}(x)=$ $1, p_{f}(x)=0$, using $g_{p}=0.01$

Reducing the value of the surface energy parameter $g_{p}$ from $g_{p}=0.1$ to $g_{p}=0.01$ leads to a mitigation of the polymer preferring behavior and thus thinner polymer and fullerene layers at the bottom of the domain, see Figure 11. Interchanging the values of the space-dependent functions $p_{i}(x)$ in (13) via $p_{p}(x)=0$ and $p_{f}(x)=1$ for all $x \in \Gamma_{b}$ simply leads to an exchange in the roles of polymer and fullerene at the substrate.

Finally switching off both surface energy functions, i.e., $p_{p}(x)=0$ and $p_{f}(x)=0$ for all $x \in \Gamma_{b}$, leads to a preference of solvent at the bottom boundary and thus to a slim layer of pure solvent in this region. In contrast to the previous results one observes the formation of several thin layers of polymer and fullerene moving from the substrate upwards (see Figure 12). As time progresses these layers successively coalesce with the bulk region. Additionally one observes a transition region at the top boundary, where the solvent concentration $\phi_{s}$ is increasing, whereas the system in the previous examples did not contain any solvent any more. Experimenting with different combinations of parameters in some cases led to unexpected results, such as the concentrations significantly exceeding the defined interval of $[0,1]$. Based on the available data it is not possible to decide, whether these issues were caused by numerical errors, physically insensible parameter constellations or inadequacies in the mathematical model.

Overall, due to the missing availability, the validation 
$\begin{array}{llllll}1.0 & 0.8 & 0.6 & 0.4 & 0.2 & 0\end{array}$
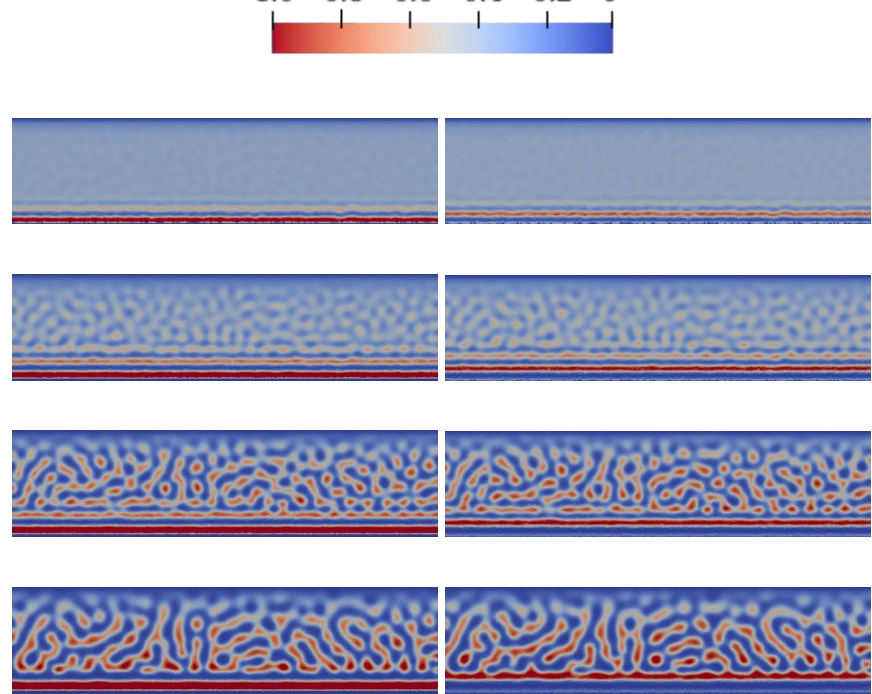

Figure 12: Morphology evolution for the preference of solvent at the substrate, i.e., $p_{p}(x)=p_{f}(x)=0$

of the achieved simulation results was only possible through qualitative comparison with results from the literature, not by comparison with actual experimental data from the real world. Nevertheless, the results presented are qualitatively comparable to [12].

\section{Conclusion}

This work gives an introduction to the field of organic solar cells and a mathematical description using a phase field model. The derivation of chemical potential terms using the Flory-Huggins theory allows the formulation of the manufacturing process as a gradient flow along the chemical potential. The derivation of the weak form of an initial boundary value problem tailored for the application of organic solar cells enables an implementation using the finite element method. Although the obtained results could not have been verified using real experimental data, qualitatively similar results to the literature have been presented. This is especially true for the influence of the surface energy term modeling a differing pattern formation of the active layer at the substrate.

Over the course of the work, several simplifications have been made. The inclusion of a stable implementation of the logarithmic potential term as well as two additional terms included in the mathematical model in [12], describing stochastic noise and an explicit height tracking of the film, should improve the model further. In addition, the implementation of tailored numerical techniques such as time-stepping or adaptivity, taking care of the strongly inhomogeneous distribution of con- centration gradients in the domain towards the end of the simulation, could provide more accurate results.

Code Availability: The source code of the implementations used to compute the presented results is available under the

$$
\text { doi: } 10.5281 / \text { zenodo.3228202 }
$$

\section{References}

[1] https://fenics.readthedocs.io/projects/dolfin/en/2017.2.0/demos/ cahn-hilliard/python/demo_cahn-hilliard.py.html (accessed on $05 / 05 / 2019$ ).

[2] https://www.nrel.gov/pv/assets/pdfs/best-research-cellefficiencies-190416.pdf (accessed on 05/05/2019).

[3] J. Bosch. Fast Iterative Solvers for Cahn-Hilliard Problems. PhD thesis, Otto-von-Guericke Universität Magdeburg, 2016.

[4] J. Bosch and M. Stoll. Preconditioning for Vector-Valued CahnHilliard Equations. SIAM Journal on Scientific Computing, 37 (5):S216-S243, 2015. doi: 10.1137/14M0973633.

[5] B. Burger. Stromerzeugung in Deutschland im Jahr 2017. Fraunhofer ISE, Download from www.ise.fraunhofer.de, version of 08/05/2018.

[6] C. M. Elliott and H. Garcke. On the Cahn-Hilliard Equation with Degenerate Mobility. SIAM J. Math. Anal., 27 (2):404-423, March 1996. ISSN 0036-1410. doi: 10.1137/ S0036141094267662.

[7] P. J. Flory. Principles of Polymer Chemistry. Cornell University Press, 1953.

[8] R. A. L. Jones and W. R. Randal. Polymers at Surfaces and Interfaces. Cambridge University Press, 1999. ISBN 9780521479653. doi: 10.1017/CBO9780511623196.

[9] D. Raabe. Computational Materials Science. 1998. doi: 10. $1002 / 3527601945$.

[10] L. M. Robeson. Polymer Blends. Carl HanserVerlag, 2007. doi: 10.3139/9783446436503.

[11] H. Wirth. Aktuelle Fakten zur Photovoltaik in Deutschland. Fraunhofer ISE, Download from www.pv-fakten.de, version of 25/10/2018.

[12] O. Wodo and B. Ganapathysubramanian. Modeling morphology evolution during solvent-based fabrication of organic solar cells. Computational Materials Science, 55:113-126, 2012. ISSN 0927-0256. doi: 10.1016/j.commatsci.2011.12.012. 